\title{
Pharmacological Treatment of Severe Breathing Abnormalities in a Case of HNRNPU Epileptic Encephalopathy
}

\author{
Carlotta Spagnolia Susanna Rizzi ${ }^{a} \quad$ Grazia Gabriella Salerno ${ }^{a} \quad$ Daniele Frattini ${ }^{a}$ \\ Juha Koskenvuo ${ }^{b}$ Carlo Fusco ${ }^{a, c}$ \\ ${ }^{a}$ Child Neurology Unit, Department of Pediatrics, Azienda USL-IRCCS di Reggio Emilia, Reggio Emilia, Italy; \\ bBlueprint Genetics, Helsinki, Finland; 'Pediatric Neurophysiology Laboratory, Azienda USL-IRCCS di Reggio Emilia, \\ Reggio Emilia, Italy
}

\section{Established facts}

- HNRNPU pathogenic variants cause early infantile epileptic encephalopathy type 54 .

- Associated abnormal breathing patterns have been described only once.

\section{Novel insights}

- We report a patient with severe paroxysmal hyperventilation, apnea, and syncope.

- Breathing abnormalities were reminiscent of Pitt-Hopkins and Rett syndrome.

- Acetazolamide, alprazolam and aripiprazole led to significant improvement.

\section{Keywords}

HNRNPU · Epileptic encephalopathy · Hyperventilation · Apnea · Therapy · Pitt-Hopkins syndrome

\section{Abstract \\ Abnormal breathing patterns are a typical feature of Rett and Pitt-Hopkins syndrome and their variants. Their treat- ment can be challenging, with a risk of long-term detrimen- tal consequences. Early infantile epileptic encephalopathy (EIEE) type 54 is a rare epileptic encephalopathy caused by pathogenic variants in the heterogeneous nuclear ribonu- cleoprotein $U$ (HNRNPU) gene. Only one case has been}

described in the literature with episodes of hyperventilation and apnea, but treatment was not discussed. We describe the clinical and genetic features and treatment strategies in a case of EIEE type 54 and severely abnormal breathing pattern. A novel and likely pathogenic c.2277dup, p.(Pro760Serfs*5) variant in the HNRNPU gene was found in a male patient with severe episodes of hyperventilation and apnea, leading to syncope. Combination therapy with acetazolamide, alprazolam and aripiprazole led to significant clinical improvement. Although HNRNPU has not been implicated in breathing control, pathogenic variants in this gene can be associated with the development of abnormal breathing patterns reminiscent of Rett and Pitt-Hopkins 
syndrome. Its function as a gene expression regulator and its interaction with transcription factors offers a potential pathogenetic link between these 3 disorders. Based on our experience, treatment strategies can be similar to those already applied for patients with Pitt-Hopkins and Rett syndrome.

(c) 2021 S. Karger AG, Basel

Genetic syndromes with abnormal breathing patterns include Rett and Rett-like, Pitt-Hopkins and Pitt-Hopkins-like phenotypes. Breath-holding, apnea, hyperventilation, and interchange between these abnormal respiratory rhythms are most commonly described. Underlying pathophysiologic mechanisms are complex, and treatment and management can often prove challenging, but can become imperative in severe cases in order to contrast secondary oxidative stress and cerebral hypoxic-ischemic changes [Mackay et al., 2017].

The HNRNPU gene encodes the heterogeneous nuclear ribonucleoprotein (hnRNP) U, a nuclear phosphoprotein taking part in the spliceosome, binding to pre-mRNA in vivo and possibly involved in pre-mRNA splicing [Ye et al., 2015]. It has an important role in fetal brain development, and is expressed in adult kidney, heart and brain [Bramswig et al., 2017].

De novo heterozygous HNRNPU pathogenic variants cause early infantile epileptic encephalopathy (EIEE) type 54 (OMIM 617391), characterized by early-onset (frequently refractory) seizures, severe intellectual disability with severe speech impairment, hypotonia, and often central nervous system (CNS), heart and kidney abnormalities, possibly associated with dysmorphisms and abnormal growth parameters [Bramswig et al., 2017]. Behavioral issues can be present, and some patients display autistic features with stereotypies [Bramswig et al., 2017; Depienne et al., 2017; Leduc et al., 2017].

Here, we describe a patient carrying a novel heterozygous, likely pathogenic variant in the HNRNPU gene, who presented with pharmacoresistant epileptic encephalopathy, severe developmental delay with absent speech, and episodes of severe hyperventilation with apnea and syncope. We will discuss therapeutic choices made for our patient and compare them to the literature.

\section{Case Report}

Our male patient, now aged 10, was born at term after caesarean section (CS) (previous CS) with appropriate for age birth weight and normal Apgar scores. His mother has a diagnosis of schizophrenia and was on valproate and olanzapine during the first 2 months of pregnancy.

Developmental milestones were severely delayed, with onset of independent walking at 36 months and absent speech. Since infancy, macrocephaly, hypotonia and dysmorphisms (hypertelorism, depressed nasal bridge, broad nasal tip, and full lips) were apparent. His current behavioral profile shows significant autistic features and hand stereotypies (clapping).

$\mathrm{He}$ had his first febrile seizure at 7 months of age. One month later, recurrent afebrile epileptic episodes ensued during sleep (staring, tonic version to the right, oxygen desaturation with cyanosis, upper limbs automatisms), and he was started on carbamazepine.

In the following 4 years, he continued to experience focal seizures (motor automatisms, drooling, staring, and at times clonic jerks) with dysautonomic components (desaturation, cyanosis, pallor, and sometimes apnea), which proved refractory to carbamazepine, zonisamide, and topiramate, alone or in association. At 5 years of age, seizures with upper limbs abduction and loss of postural tone also began (multiple episodes per day). Levetiracetam was initially added to topiramate, and then topiramate was withdrawn due to lack of efficacy. At 6 years of age, clobazam was first added to levetiracetam, and then also valproate. With this polytherapy, seizure frequency progressively reduced, and the patient was seizure free by 8 years of age. Background EEG is characterized by excess slow over the temporal regions bilaterally and by multifocal interictal paroxysmal discharges.

In addition to stereotypies, repeated vocalizations and episodes of hyperpnea and apnea were documented since early childhood during wakefulness, but exacerbated significantly at 9 years and a half, when he presented multiple episodes per day, characterized by hyperventilation, apnea, cyanosis, sometimes followed by syncope, with severe oxygen desaturation (minimum documented: $30 \%$ ). The patient was acutely admitted to hospital and EEG ruled out an epileptic origin of these episodes. ECG confirmed a grade 1 atrioventricular block.

Acetazolamide was started at $250 \mathrm{mg} /$ day, with improvement of the severity of these episodes. Aripiprazole ( $5 \mathrm{mg} /$ day) and alprazolam $(0.25 \mathrm{mg} /$ day $)$ were added, with further reduction in both frequency and severity (minimum oxygen saturation: 70\%). At admission, our patient was experiencing subcontinuous episodes of hyperventilation followed by apnea, facial pallor and lip cyanosis, sometimes with balance impairment and at times progressing to syncope and falling to the floor, each episode lasting less than $60 \mathrm{~s}$ with free intervals lasting between $1 \mathrm{~min}$ and a half and 3 min during wakefulness. After introducing acetazolamide, episodes reduced to a maximum of 5-6 per hour during wakefulness, with minimal saturation of oxygen of $55-60 \%$ (usually around $70 \%$ ) and quicker resolution. After adding aripiprazole, 4-5 second-long episodes (multiple per day) persisted, with immediate recovery, which by adding alprazolam further reduced in frequency and severity (minimum saturation of oxygen: 77\%, distractibility, no syncope), allowing safe discharge home after 13 days since admission.

During follow-up, many diagnostic investigations were performed, including negative brain MRI, normal auditory brainstem potentials, normal karyotype, unremarkable single gene testing and non-contributing next-generation sequencing panel targeted for epilepsy and epileptic encephalopathies. 
Unremarkable neurometabolic investigations include: lactate, ammonia, lactate/pyruvate ratio, urinary and plasmatic amino acids, urinary organic acids, VLCFA, lysosomal enzymes (alpha-galactosidase, ARA A and ARA B, beta-exosaminidase, fucosidase).

By whole-exome sequencing, we documented the likely pathogenic c.2277dup, p.(Pro760Serfs*5) variant in the HNRNPU gene. The variant has not been observed in large reference population cohorts of the Genome Aggregation database (gnomAD). It duplicates one base pair, generating a frameshift leading to a premature stop codon at position 5 in a new reading frame. It is predicted to cause loss of normal protein function, either through protein truncation or nonsense-mediated mRNA decay. To our knowledge, it has not been reported in disease-related variation databases (ClinVar, HGMD) nor published in the literature. Segregation analysis was not possible; parents were unavailable.

\section{Discussion and Conclusion}

Microdeletions in the 1q44 region encompassing HNRNPU have been described in patients with ID, also displaying seizures, microcephaly, and corpus callosum abnormalities. HNRNPU, a key player in brain development [Poot, 2019], is considered as the major determinant of epilepsy in these patients [Depienne et al., 2017].

Apart from gross deletions/insertions, de novo loss of function and missense variants have later been described, with small intragenic deletions and duplications being documented sporadically [Bramswig et al., 2017]. HNRN$P U$ exhibits haploinsuficiency and is intolerant to loss-offunction mutations (haploinsufficiency index: $7.65 \%$, pLI score: 1.00; https://decipher.sanger.ac.uk).

The HNRNPU protein is located in the nucleus and binds to its matrix, helping localize genes and proteins (including transcription factors within the nucleus) and facilitates gene, mRNA, and protein interactions during mRNA processing, thus regulating gene expression [Leduc et al., 2017; Poot, 2019].

Specific features of breathing are controlled by different components of the respiratory network, which involves the ventrolateral medulla, especially the preBoetzinger complex, as the key generator of the hypoxic response. Reciprocal inhibition between 2 groups of neurons ("early inspiratory" and "postinspiratory") located in the brainstem is the primary determinant of respiratory rhythm. However, more rostral areas also have an influence and can be modulated by environmental and internal conditions (i.e., anxiety) [Ramirez et al., 2013].

Appropriate termination of inspiratory cycle is the main regulator of breathing cycles and is actively determined by serotoninergic neurons. Abnormal maturation and modulation of neurons involved in respiratory rhythm generators, resulting in imbalances in synaptic transmission and alterations in neuromodulatory transmission, including GABAergic and brain-derived neurotrophic factor signaling have been hypothesized in the pathogenesis of breathing abnormalities in Rett and PittHopkins syndrome [Ramirez et al., 2013]. Moreover, reduced levels of biogenic amines (dopamine, serotonin, and norepinephrine) and their metabolites have been documented in postmortem brain tissues of Rett syndrome patients [Lekman et al., 1989]. Of note, both MECP2 (causing Rett syndrome) [MacKay et al., 2017] and TCF4 (causing Pitt-Hopkins syndrome) [Verhulst et al., 2012] are involved in gene transcription and regulation of gene expression, and HNRNPU is known to interact with transcription factors within the nucleus [Kiledjian and Dreyfuss, 1992], thus suggesting a possible involvement of common pathways, even if (to the best of our knowledge) there is no current publication exploring the HNRNPU role in the genesis of breathing patterns.

While autistic features and stereotypies (including hand clapping and flapping) have been repeatedly reported [Bramswig et al., 2017; Depienne et al., 2017; Leduc et al., 2017; Shimada et al., 2018], including nonspecified self-stimulatory behaviors [Leduc et al., 2017], there is only one previous description of a 5-year-old boy with a frameshift $H N R N P U$ variant displaying autistic behavior and "Rett-like" features, including respiratory abnormalities with episodes of apnea and hyperpnea followed by falling, findings which are strikingly similar to the ones we documented in our patient. However, the authors do not discuss therapeutic management [Shimada et al., 2018]. Therefore, our treatment choices were based on available evidence and clinical practice in the treatment of abnormal breathing patterns in children with PittHopkins and Rett syndrome.

Acetazolamide administration in our patient resulted in a decrease in the severity of his attacks. Acetazolamide improves hyperventilation and apneic episodes not only by carbonic anhydrase inhibition (which increases the apneic threshold through loss of bicarbonate and consequent metabolic acidosis), but also with direct effect on chemoreceptors [Swenson and Hughes, 1993], which stabilizes breathing patterns. Its efficacy has been documented both in Pitt-Hopkins and Rett syndrome [Verhulst et al., 2012; Mackay et al., 2017]. We prescribed alprazolam in order to modulate GABA-mediated synaptic action [Ramirez et al., 2013]. Finally, aripiprazole (an atypical antipsychotic, with affinity for both dopaminergic and serotoninergic receptors) was associated 


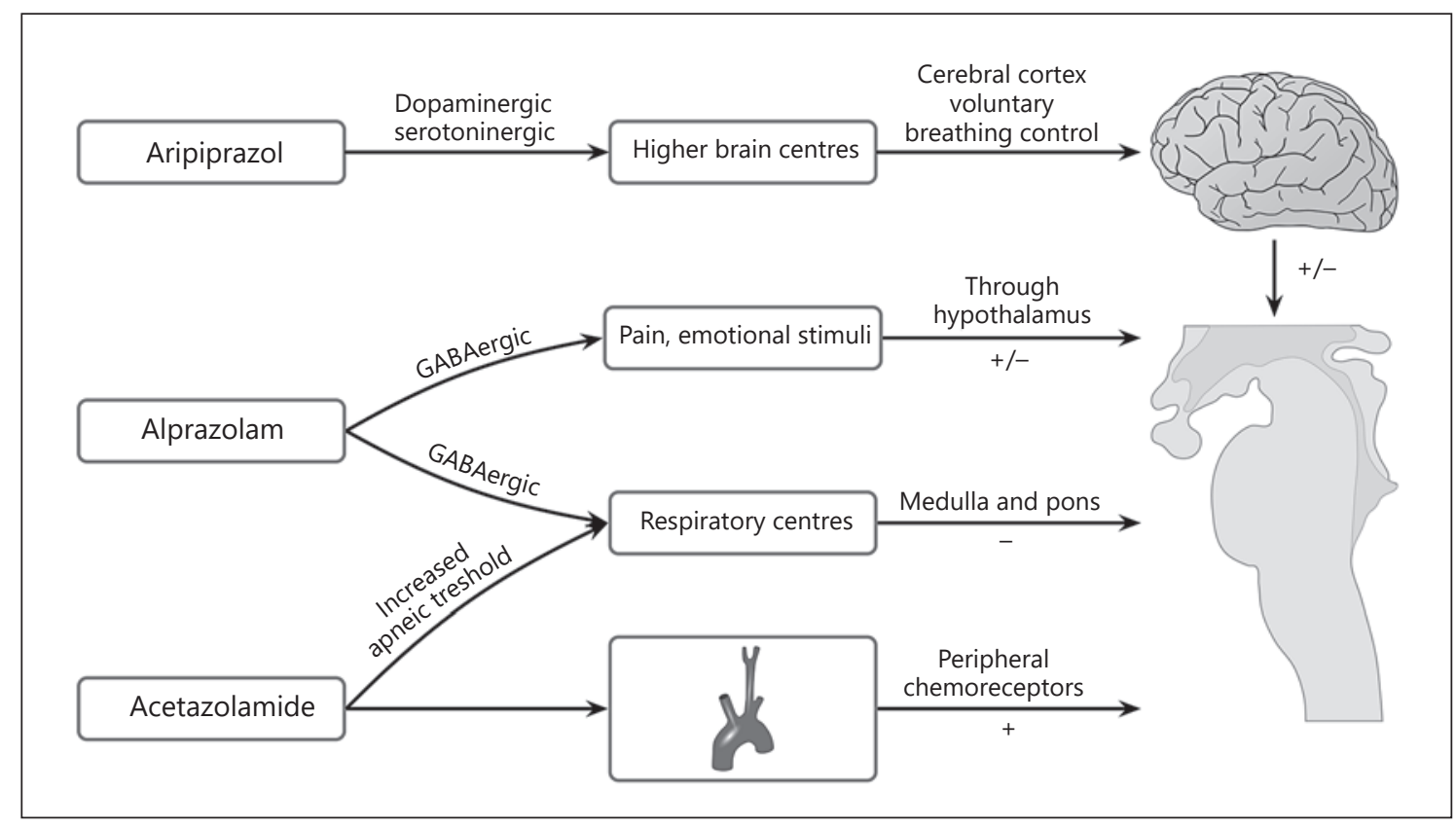

Fig. 1. Neural control of breathing and main sites of action of the drugs administered in our patient. +, excitatory action; -, inhibitory action; +/-, complex modulation.

based on its efficacy in reducing stereotypies and compulsive behaviors, also documented in children with autism spectrum disorders [Hirsch and Pringsheim, 2016] (Fig. 1). This combination therapy led to significant clinical improvement and prevention of syncopal episodes, allowing safe discharge home. The clinical need to quickly proceed to a combination therapy reduces our ability to disentangle specific contributions of each medication in achieving our therapeutic goal.

Finally, during fetal development, our patient was exposed to valproate, which is well-known to cause dosedependent teratogenicity. These detrimental effects are known as the "valproate fetal syndrome" and include neural tube defects, cardiovascular abnormalities, genitourinary defects (cryptorchidism, hypospadia), musculoskeletal malformations (vertebral/ribs fusion, agenesis or duplication, contractions of the small joints, long overlapping fingers, foot deformities, nail abnormalities) and craniofacial defects with dysmorphisms (cleft lip and palate, epicanthal folds, infraorbital crease or groove, flat nasal bridge, small nose with anteverted nostrils, long upper lip with shallow philtrum, small mouth with downturned angles, thin upper vermilion border), endocrinological disorders, developmental delay and intellectual disability, and autism [Mutlu-Albayrak et al., 2017]. Although we cannot exclude a contribution of valproate teratogenicity in the overall clinical phenotype of our patient, the major malformations caused by fetal exposure to valproate are absent, and we believe his core clinical characteristics can be best explained by his HNRNPU variant [Bramswig et al., 2017; Leduc et al., 2017].

In conclusion, we describe a patient with EIEE type 54 due to a novel likely pathogenic variant in the HNRNPU gene, who experienced severe episodes of hyperventilation and apnea with falling, which improved on a combination therapy with acetazolamide, alprazolam and aripiprazole. Thus, we described abnormal breathing episodes in this condition, an association only reported once in the literature, and reported for the first time on its pharmacological treatment.

\section{Statement of Ethics}

The authors have no ethical conflicts to declare. Investigations were conducted in line with the principles of the Declaration of Helsinki. The paper is exempt from ethical committee approval: Institutional review board approval was not required for this type of publication. Informed consent was obtained from the legal guardian. 


\section{Conflict of Interest Statement}

The authors C.S., S.R., D.F., G.G.S., and C.F. have no conflicts of interest to declare. J.K. is the Laboratory Director and Chief Medical Officer of Blueprint Genetics.

\section{Funding Sources}

No funding was secured for the completion of this study.

\section{Author Contributions}

Conceptualization: C.S., C.F. Writing and original draft preparation: C.S. Clinical data collection: D.F., C.S. Molecular genetic data: J.K. Critical review: S.R., G.G.S., D.F., J.K., C.F. All authors analyzed and interpreted the data and approved the manuscript in its final form.

\section{References}

Bramswig NC, Lüdecke HJ, Hamdan FF, Altmüller J, Beleggia F, Elcioglu NH, et al. Heterozygous HNRNPU variants cause early onset epilepsy and severe intellectual disability. Hum Genet. 2017;136(7):821-34.

Depienne C, Nava C, Keren B, Heide S, Rastetter A, Passemard S, et al. Genetic and phenotypic dissection of 1q43q44 microdeletion syndrome and neurodevelopmental phenotypes associated with mutations in ZBTB18 and HNRNPU. Hum Genet. 2017;136(4):463-79.

Hirsch LE, Pringsheim T. Aripiprazole for autism spectrum disorders (ASD). Cochrane Database Syst Rev. 2016(6):CD009043.

Kiledjian M, Dreyfuss G. Primary structure and binding activity of the hnRNP $U$ protein: binding RNA through RGG box. EMBO J. 1992;11(7):2655-64.

Leduc MS, Chao HT, Qu C, Walkiewicz M, Xiao $\mathrm{R}$, Magoulas P, et al. Clinical and molecular characterization of de novo loss of function variants in HNRNPU. Am J Med Genet A. 2017;173(10):2680-9.
Lekman A, Witt-Engerström I, Gottfries J, Hagberg BA, Percy AK, Svennerholm L. Rett syndrome: Biogenic amines and metabolites in postmortem brain. Pediatr Neurol. 1989;5(6): 357-62.

Mackay J, Downs J, Wong K, Heyworth J, Epstein A, Leonard H. Autonomic breathing abnormalities in Rett syndrome: caregiver perspectives in an international database study. J Neurodev Disord. 2017;9:15.

Mutlu-Albayrak H, Bulut C, Çaksen H. Fetal Valproate Syndrome. Pediatr Neonatol. 2017; 58(2):158-64.

Poot M. HNRNPU: key to neurodevelopmental disorders such as intellectual delay, epilepsy, and autism. Mol Syndromol. 2019;9(6):275-

Ramirez JM, Ward CS, Neul JL. Breathing challenges in Rett syndrome: lessons learned from humans and animal models. Respir Physiol Neurobiol. 2013;189(2):280-7.
Shimada S, Oguni H, Otani Y, Nishikawa A, Ito S, Eto K, et al. An episode of acute encephalopathy with biphasic seizures and late reduced diffusion followed by hemiplegia and intractable epilepsy observed in a patient with a novel frameshift mutation in HNRNPU. Brain Dev. 2018;40(9):813-8.

Swenson ER, Hughes JM. Effects of acute and chronic acetazolamide on resting ventilation and ventilatory responses in men. J Appl Physiol. 1993;74(1):230-7.

Verhulst SL, De Dooy J, Ramet J, Bockaert N, Van Coster R, Ceulemans B, et al. Acetazolamide for severe apnea in Pitt-Hopkins syndrome. Am J Med Genet A. 2012;158A(4):932-4.

Ye J, Beetz N, O'Keeffe S, Tapia JC, Macpherson $\mathrm{L}$, Chen WV, et al. hnRNP U protein is required for normal pre-mRNA splicing and postnatal heart development and function. Proc Natl Acad Sci USA. 2015;112(23): E3020-9. 